\title{
On-Demand Single Photons with High Extraction Efficiency and Near-Unity Indistinguishability from a Resonantly Driven Quantum Dot in a Micropillar
}

Ding, Xing ; He, Yu; Duan, Z.-C.; Gregersen, Niels; Chen, M.-C.; Unsleber, S.; Maier, Sebastian; Schneider, Christian; Kamp, Martin; Höfling, Sven

Total number of authors:

12

Published in:

Physical Review Letters

Link to article, DOI:

10.1103/PhysRevLett.116.020401

Publication date:

2016

Document Version

Publisher's PDF, also known as Version of record

Link back to DTU Orbit

Citation (APA):

Ding, X., He, Y., Duan, Z-C., Gregersen, N., Chen, M-C., Unsleber, S., Maier, S., Schneider, C., Kamp, M. Höfling, S., Lu, C-Y., \& Pan, J-W. (2016). On-Demand Single Photons with High Extraction Efficiency and NearUnity Indistinguishability from a Resonantly Driven Quantum Dot in a Micropillar. Physical Review Letters, 116, [020401]. https://doi.org/10.1103/PhysRevLett.116.020401

\section{General rights}

Copyright and moral rights for the publications made accessible in the public portal are retained by the authors and/or other copyright owners and it is a condition of accessing publications that users recognise and abide by the legal requirements associated with these rights.

- Users may download and print one copy of any publication from the public portal for the purpose of private study or research.

- You may not further distribute the material or use it for any profit-making activity or commercial gain

- You may freely distribute the URL identifying the publication in the public portal 


\title{
On-Demand Single Photons with High Extraction Efficiency and Near-Unity Indistinguishability from a Resonantly Driven Quantum Dot in a Micropillar
}

\author{
Xing Ding, ${ }^{1,2,3}$ Yu He, ${ }^{1,2,3}$ Z.-C. Duan, ${ }^{1,2,3}$ Niels Gregersen, ${ }^{4}$ M.-C. Chen, ${ }^{1,2,3}$ S. Unsleber, ${ }^{5}$ S. Maier, ${ }^{5}$ \\ Christian Schneider, ${ }^{5}$ Martin Kamp, ${ }^{5}$ Sven Höfling, ${ }^{1,5,6}$ Chao-Yang Lu, ${ }^{1,2,3, *}$ and Jian-Wei Pan ${ }^{1,2,3, \uparrow}$ \\ ${ }^{1}$ Shanghai Branch, National Laboratory for Physical Sciences at Microscale and Department of Modern Physics, \\ University of Science and Technology of China, Shanghai, 201315, China \\ ${ }^{2}$ CAS Center for Excellence and Synergetic Innovation Center in Quantum Information and Quantum Physics, \\ University of Science and Technology of China, Hefei, Anhui 230026, China \\ ${ }^{3}$ CAS-Alibaba Quantum Computing Laboratory, Shanghai 201315, China \\ ${ }^{4}$ DTU Fotonik, Department of Photonics Engineering, Technical University of Denmark, \\ Building 343, DK-2800 Kongens Lyngby, Denmark \\ ${ }^{5}$ Technische Physik, Physikalisches Instität and Wilhelm Conrad Röntgen-Center for Complex Material Systems, \\ Universitat Würzburg, Am Hubland, D-97074 Wüzburg, Germany \\ ${ }^{6}$ SUPA, School of Physics and Astronomy, University of St. Andrews, St. Andrews KY16 9SS, United Kingdom
}

(Received 29 September 2015; published 14 January 2016)

\begin{abstract}
Scalable photonic quantum technologies require on-demand single-photon sources with simultaneously high levels of purity, indistinguishability, and efficiency. These key features, however, have only been demonstrated separately in previous experiments. Here, by $s$-shell pulsed resonant excitation of a Purcellenhanced quantum dot-micropillar system, we deterministically generate resonance fluorescence single photons which, at $\pi$ pulse excitation, have an extraction efficiency of $66 \%$, single-photon purity of $99.1 \%$, and photon indistinguishability of $98.5 \%$. Such a single-photon source for the first time combines the features of high efficiency and near-perfect levels of purity and indistinguishabilty, and thus opens the way to multiphoton experiments with semiconductor quantum dots.
\end{abstract}

DOI: 10.1103/PhysRevLett.116.020401

Single-photon devices [1] that deterministically emit one and only one photon at a time are central resources for scalable photonic quantum technologies [2]. In particular, they are of considerable interest in boson sampling [3], an intermediate quantum computation where it is estimated that with 20-30 single photons one can demonstrate complex tasks that can be difficult for classical computers. To be useful for these applications, it is crucial that the single-photon source simultaneously possesses high efficiency, near perfect photon antibunching and indistinguishability-a long sought-after goal in quantum photonics.

Self-assembled quantum dots (QDs) [4] have been shown to possess the highest quantum efficiency in all solid-state single-photon devices so far, and thus are promising as deterministic single-photon emitters. Tremendous progress has been made in the past decades in demonstrations of various single-photon sources [5-12]; however, none has allowed a scalable extension to multiphoton experiments. The reason is that most previous experiments either relied on nonresonant excitation of a QD microcavity that degraded the photon purity and indistinguishability, or used resonant excitation of a QD in a planar cavity that limited the extraction efficiency.

In the nonresonant excitation experiments, single-photon generation efficiencies typically increased asymptotically with pump power, and a trade-off between efficiency and single-photon purity and/or indistinguishability were observed [5-10]. It has been shown that the recapture of carriers into the QD micropillar due to nonresonant pumping can lead to a significant degradation of the single-photon purity [13]. Furthermore, the photon's indistinguishability can be reduced by nonresonant excitation that induces homogeneous broadening of the excited state [14] and uncontrolled emission time jitter from the nonradiative high-level to $s$-shell relaxation [15]. The detrimental effect of the time jitter can be more severe when using a QD-microcavity system where the Purcell-reduced radiative lifetime $\left(T_{1}\right)$ of the $\mathrm{QD}$, which bounds the single photon's coherence time $\left(T_{2}\right)$ by $T_{2} \leq 2 T_{1}$, is comparable to or smaller than the time jitter [15].

To overcome these shortcomings, increasing efforts have been devoted to resonant excitation of QDs [11,12,16-18]. By $s$-shell resonant laser excitation on a single QD with picosecond laser pulses, near-background-free resonance fluorescence (RF) has been obtained and allowed the observation of Rabi oscillations [11]. Under $\pi$ pulse excitation, single photons have been deterministically generated with a single-photon purity of $98.8(2) \%$ and indistinguishability of $97(2) \%$. However, the planar cavity structure used in Ref. [11] has allowed only $~ 6 \%$ of the generated single photons collected by the first lens.

In this Letter, we report the first single-photon source that combines near perfect single-photon purity, indistinguishability, and high extraction efficiency. By pulsed strict 

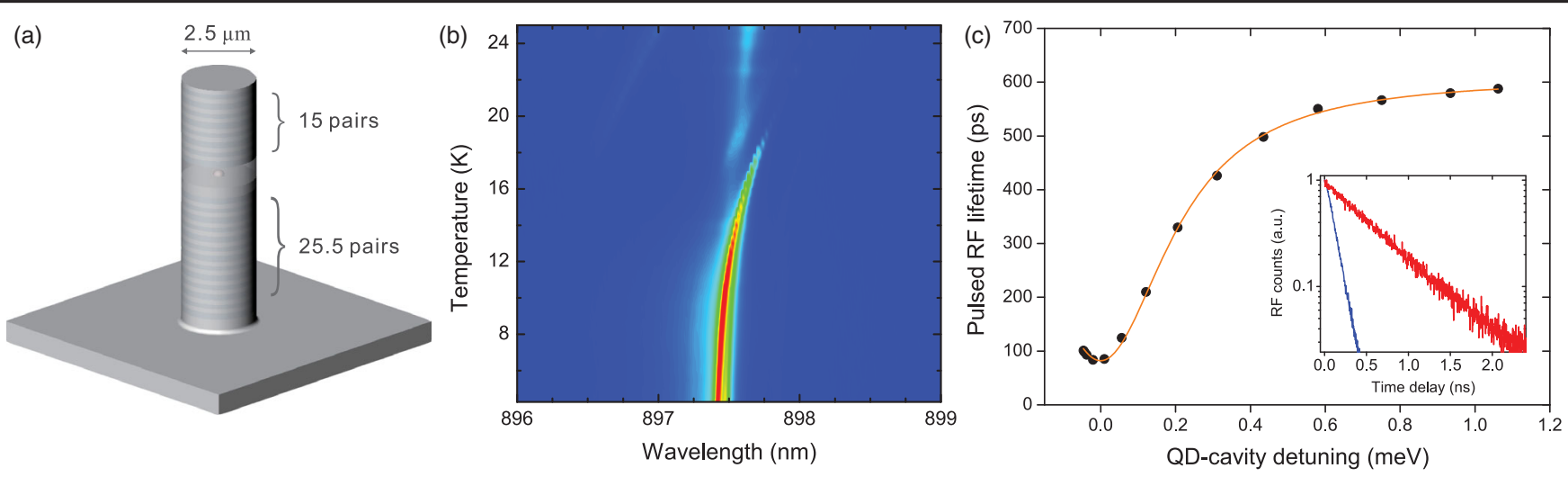

FIG. 1. Purcell-enhanced QD-micropillar system. (a) An illustration of a single QD embedded in a micropillar. The QD is grown via molecular beam epitaxy, embedded in a $\lambda$-thick GaAs cavity and sandwiched between 25.5 lower and 15 upper DBR stacks. Micropillars with $2.5 \mu \mathrm{m}$ diameter were defined via electron beam lithography [18]. (b) 2D intensity (in log scale) plot of temperaturedependent microphotoluminescence spectra. The excitation cw laser is at $780 \mathrm{~nm}$ wavelength and the power is $\sim 3 \mathrm{nW}$. (c) Pulsed RF lifetime as a function of QD-cavity detuning by varying the temperature. The time-resolved data are measured using a superconducting nanowire single-photon detectors with a fast time resolution of $\sim 63 \mathrm{ps}$. The orange curve is a fit using the standard theoretical formula from Ref. [19]. The inset shows two examples of time-resolved RF counts at QD-cavity resonance and at far detuning.

resonant excitation of a Purcell-enhanced QD-micropillar system, at $\pi$ pulse we obtain pulsed RF single photons with a count rate of $3.7 \times 10^{6} / \mathrm{sec}$ on a silicon single-photon detector, which corresponds to an extraction efficiency of $\sim 66 \%$ from GaAs. The RF photons show an antibuching of $g^{2}(0)=0.009(2)$ and a raw (corrected) two-photon interference visibility of 96.4(3)\% [98.5(4)\%]. Our work opens the way to scalable multiphoton quantum information experiments with solid-state devices.

Our experiments were performed on an InAs/GaAs selfassembled QD embedded inside a $2.5 \mu \mathrm{m}$ diameter micropillar cavity which has 25.5 (15) $\lambda / 4$-thick AlAs/GaAs mirror pairs forming the lower (upper) distributed Bragg reflectors [18] [see Fig. 1(a)]. The device is cooled inside a cryogen-free bath cryostat with a temperature that can be finely tuned from 4.2 to $30 \mathrm{~K}$. We first characterize the QD through microphotoluminescence measurements with $780 \mathrm{~nm}$ laser excitation. Figure 1(b) shows photoluminescence spectra of a single QD as a function of temperature tuning. The photoluminescence intensity reaches a plateau at a temperature range of $4.5-10 \mathrm{~K}$. The quality factor of the micropillar cavity is measured to be 6124 .

For pulsed resonant excitation, a Ti:sapphire laser is used to generate laser pulses at a central wavelength of $897.44 \mathrm{~nm}$ and a pulse width of $\sim 3 \mathrm{ps}$. The excitation laser is further filtered with an etalon with a linewidth of $45 \mathrm{GHz}$ to match the micropillar cavity. A confocal microscope is operated in a cross-polarization configuration [11], whereby a polarizer is placed in the collection arm with its polarization perpendicular to the excitation laser suppressing the laser background by a factor exceeding $10^{7}$. To determine the Purcell factor of the micropillar cavity, we perform time-resolved RF measurements for different cavity-QD detunings, as plotted in Fig. 1(c). The shortest RF lifetime is 83.9 ps at $7.8 \mathrm{~K}$ [blue curve in the inset of
Fig 1(c)]. At $28 \mathrm{~K}$ with a large ( $>1 \mathrm{meV})$ detuning, the decay time constant increases to $587.8 \mathrm{ps}$ [red curve in the inset of Fig 1(c)]. The measured lifetime as a function of detuning is well fitted by the standard weak-coupling theoretical model (orange curve) [19], which allows us to deduce a Purcell factor of 6.3(4).

Figure 2(a) shows a high-resolution spectrum of the pulsed RF, which can be best fitted using a Voigt profile with a homogeneous (Lorentzian) linewidth of 1.91(7) GHz and inhomogeneous (Gaussian) linewidth of 1.14(9) GHz. The homogeneous linewidth is very close to the lifetimelimited linewidth of $1.89 \mathrm{GHz}$. The inhomogeneous (Gaussian) component in the spectrum can be caused by spectral diffusion due to charge fluctuations in the vicinity of the QD [20].

Figure 2(b) shows the detected pulsed RF photon counts on a silicon single-photon detector as a function of incident field amplitude. We observe a Rabi oscillation which is due to coherent control of the QD two-level system [21]. The single-photon counts reaches the maximum for a $\pi$ pulse with a pumping laser power of $24 \mathrm{nW}$. We note that this is in stark contrast to previous experiments with nonresonant excitation where the generated single-photon counts typically grow asymptotically with excitation laser power; thus, a near unity efficiency would need very high power pumping.

At the $\pi$ pulse, we observe a count rate of $3700000 / \mathrm{sec}$ on a silicon single-photon detector, under $81 \mathrm{MHz}$ repetition rate laser excitation, which gives an overall system efficiency of $4.6 \%$. The signal (RF) to background (mainly laser leakage) ratio is $40: 1$. After correcting for independently calibrated photon detection efficiency $(\sim 33 \%)$, polarization extinction $(\sim 50 \%)$, transmission rate in the optical path $(\sim 60 \%$, including optical window, polarizer, and two beam splitters), and single-mode fiber coupling efficiency 
(a)

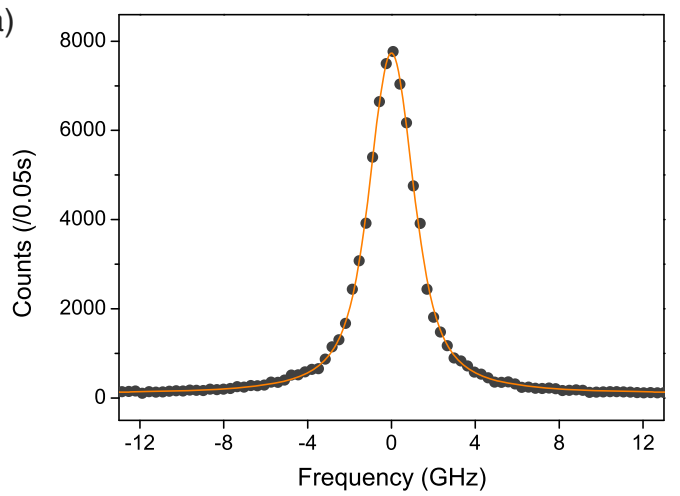

(c)

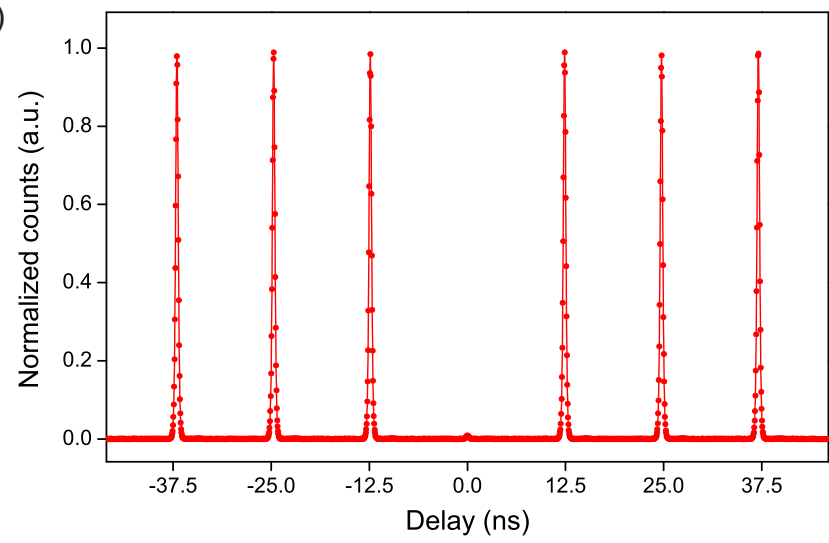

(b)

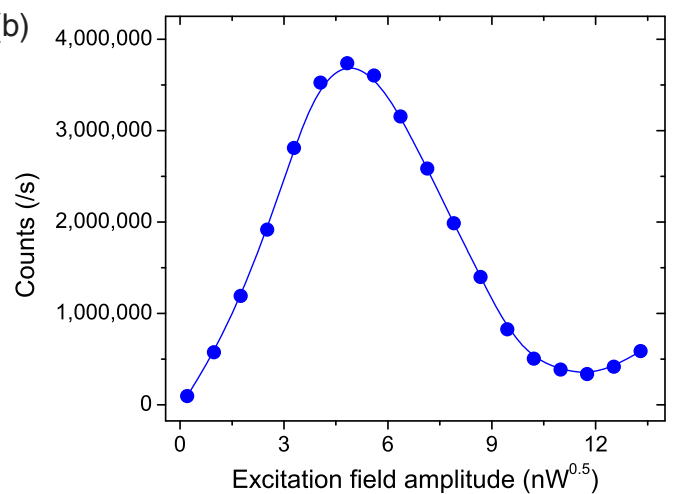

(d)

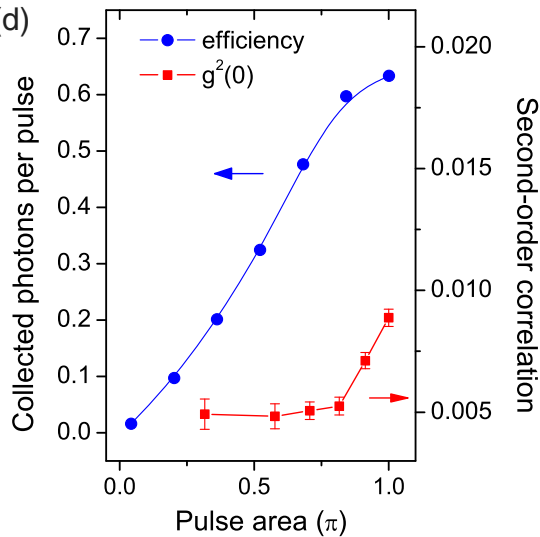

FIG. 2. Characterization of the pulsed RF single-photon source. (a) A high-resolution RF spectrum when excited by a $\pi$ pulse, obtained using a home-built Fabry-Perot scanning cavity with a finesse of 170, a linewidth of $220 \mathrm{MHz}$ (full width at half maximum), a free spectral range of $37.4 \mathrm{GHz}$, and a total transmission rate of $61 \%$. The orange line was fitted using a Voigt profile. (b) Detected pulsed RF counts on a silicon single-photon detector as a function of the square root of excitation laser power. The blue curve is a guide to the eyes. (c) Intensity-correlation histogram of the pulsed RF photons under $\pi$ pulse excitation obtained using a Hanbury Brown and Twiss-type setup [22]. The second-order correlation $g^{2}(0)=0.009(1)$ is calculated from the integrated photon counts in the zero delay peak divided by its adjacent peak. (d) The photons collected into the first lens per pulse (generation + extraction efficiency) vs singlephoton purity vs pump power.

( $\sim 72 \%)$, excited-state preparation efficiency $(\sim 96 \%)$ at $\pi$ pulse, and assuming perfect internal quantum efficiency of the QD, we estimate that $66 \%$ of the generated single photons are collected into the first objective lens $(\mathrm{NA}=0.68)$.

To further improve the signal to background ratio, we pass the RF through a 3-GHz etalon and then characterize its purity and indistinguishability. The single-photon nature of the collected RF at the $\pi$ pulse is evident from the second-order coherence measurement [22] shown in Fig. 2(c), where nearly vanishing multiphoton probability is observed at zero delay $\left[g^{2}(0)=0.009(1)\right]$. Figure 2(d) summarize the combined performance of the efficiency and single-photon purity as a function of pump power. We emphasize that the high generation and extraction efficiency are obtained with little compromise of the singlephoton purity, which is important for real applications in photonic quantum information processing.

Another crucial demand is that the photons should possess a high degree of indistinguishability, which is at the heart of optical quantum computing [2], Boson sampling [23], and solid-state quantum networks [24]. Pulsed $s$-shell resonant excitation has been demonstrated to yield a near-unity indistinguishability by eliminating emission time jitter and dephasings, however, only for QDs in planar cavities. We note that the pulsed RF technique is more critically needed for QDs with large Purcell factors where the reduced radiative lifetime approaches the time jitter $[11,15]$.

The single photons' indistinguishability is tested using non-post-selective two-photon Hong-Ou-Mandel interference [25] experiments. We first adopt a similar free-space setup as shown in Ref. [11] with a time delay of $2.1 \mathrm{~ns}$ between two pulses. Figures 3(a) and 3(b) show timedelayed histograms of normalized two-photon counts for orthogonal and parallel polarization, respectively. An almost vanishing zero-delay peak is observed for two photons with identical polarization. In contrast, for two photons with cross polarization, the zero-delay peak has the same intensity as its adjacent peaks. We obtain a raw two-photon quantum interference visibility of 0.964(3). After correcting with the residual multiphoton probability 

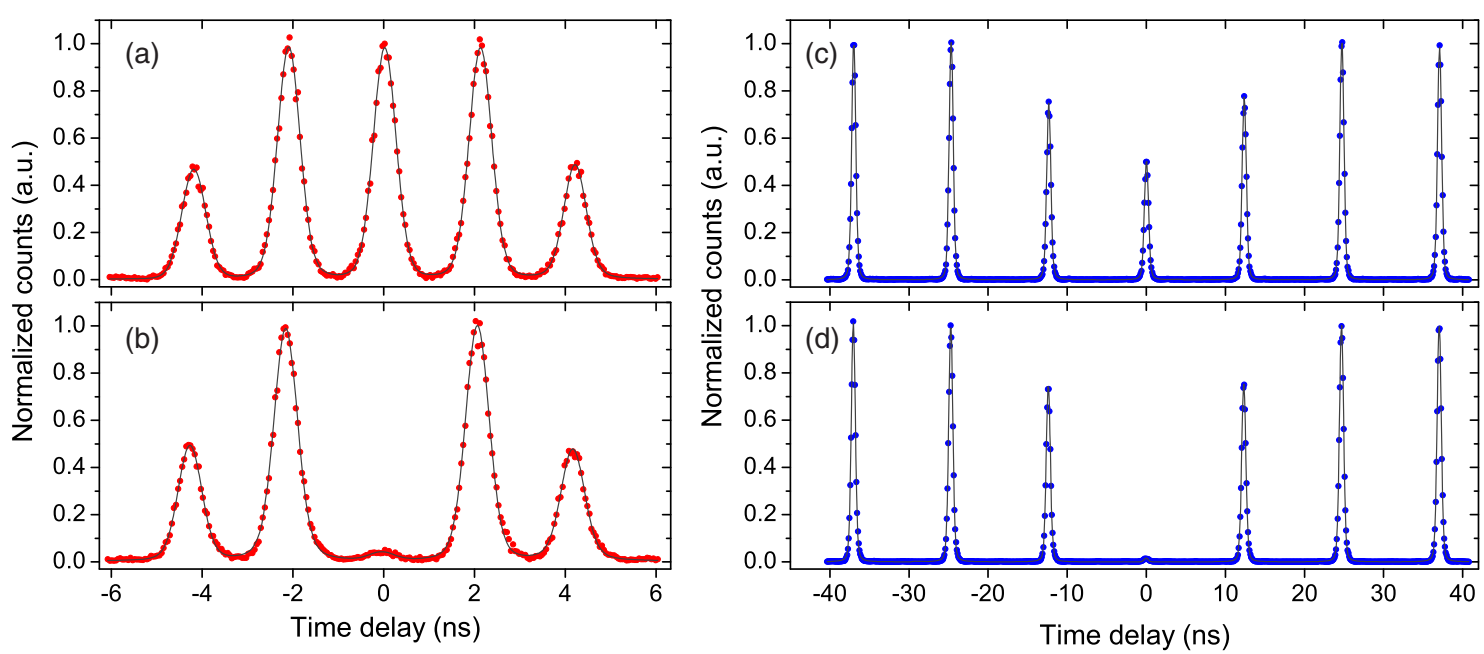

FIG. 3. Quantum interference between two pulsed RF single photons from a Purcell-enhanced QD-micropillar system. The time separation between the two single photons emitted from the single QD are set at $2.1 \mathrm{~ns}$ in (a)-(b) and 12.4 ns in (c)-(d), respectively. The input two photons are $\pi$-pulse excited and prepared in cross (a), (c) and parallel (b), (d) polarizations, respectively. The fitting function is the convolution of exponential decay (emitter decay response) with Gaussian (photon detection time response). The area of the fitted central peaks are extracted and used to calculate the raw visibility, which is 0.964(3) and 0.959(3) for the time delay of 2.1 and 12.4 ns, respectively. All the data points presented are raw data without background subtraction.

$\left[g^{2}(0)=0.009(1)\right]$, we obtained the corrected degrees of indistinguishability to be $0.985(4)$.

We further test the Hong-Ou-Mandel interference between two consecutively emitted single photons at an increased time delay of $12.4 \mathrm{~ns}$ - the laser pulse separation (see Ref. [9] for a similar setup). The resulting histograms for orthogonal and parallel polarized two photons are shown in Figs. 3(c)-3(d), from which we extract raw and corrected visibilities of 0.959(3) and 0.978(4), respectively. In strong contrast to Ref. [9] that used nonresonant excitation, increasing the time delay by $10 \mathrm{~ns}$ has a negligible effect on the photons' indistinguishability, indicating that the inhomogeneous broadening [see Fig. 2(a)] of the pulsed RF photons are mainly from spectral diffusions at time scales much slower than $10 \mathrm{~ns}$. This demonstrates the potential of this QD-micropillar device as an efficient source of a string of indistinguishable single photons, which are particularly suitable for linear optical quantum computing and Boson sampling experiments with time-bin encoding [26]. Alternatively, one can also demultiplex the single-photon string into multiple photons at separate spatial modes.

We compare the performance of the single-photon source created in this work with heralded single photons produced by parametric down conversion [27], which have served as the workhorse for multiphoton interferometric experiments in the past decades [2]. In the previous demonstration of the largest, eight-photon entanglement [28], sources of triggered single photons were generated with a count rate of $310000 / \mathrm{sec}$, multiphoton emission probability of $2.9 \%$ and raw indistinguishablility of $76 \%$, under a laser pump power of $880 \mathrm{~mW}$. For applications such as Boson sampling [23] that needs single photons as input, the single-photon source realized here is superior, as it is ten times brighter, near perfectly pure and indistinguishable, and requires a pump power that is 7 orders of magnitude lower.

In summary, by pulsed $s$-shell pumping a QDmicropillar system with a Purcell factor of 6.3, we have realized a high-performance single-photon source that at $\pi$ pulse excitation simultaneously achieves a generation efficiency of $96 \%$, extraction efficiency of $66 \%$, singlephoton purity of $99.1 \%$, and indistinguishability of $98.6 \%$. Such a single-photon source can be readily used to perform multiphoton interferometric experiments with a solid-state platform. Immediate applications include implementation of Boson sampling [23] with time-bin encoding using a loop-based architecture [26]. In addition to the photonic applications, the high-efficiency extraction of transitionselective RF would also allow a fast (nsec), high-fidelity single-shot readout of single electron spins [29].

The time-jitter free, pulsed RF method is compatible with higher Purcell factor, which can allow photon extraction to be further improved in the future by optimized microcavity fabrications, without comprising the single-photon purity and indistinguishability. The current overall system efficiency-4.6\%, the highest reported in QDs-can also be improved using techniques such as orthogonal excitation and detection of RF [16,17], near-unity-efficiency superconducting nanowire single-photon detection [30], and antireflection coatings of the optical elements.

This work was supported by the National Natural Science Foundation of China, the Chinese Academy of Sciences, and the National Fundamental Research 
Program. We acknowledge financial support by the State of Bavaria and the German Ministry of Education and Research (BMBF) within the projects Q.com-H and the Chist-era project SSQN. N. G. acknowledges support from the Danish Research Council for Technology and Production.

Note added.-After this Letter was submitted, we became aware of a related work [31].

*cylu@ustc.edu.cn †pan@ustc.edu.cn

[1] B. Lounis and M. Orrit, Rep. Prog. Phys. 68, 1129 (2005); S. Buckley, K. Rivoire, and J. Vuĉković, Rep. Prog. Phys. 75, 126503 (2012).

[2] P. Kok, W. J. Munro, K. Nemoto, T. C. Ralph, J. P. Dowling, and G. J. Milburn, Rev. Mod. Phys. 79, 135 (2007); J. L. O'Brien, A. Furusawa, and J. Vuĉković, Nat. Photonics 3, 687 (2009); J.-W. Pan, Z.-B. Chen, C.-Y. Lu, H. Weinfurter, A. Zeilinger, and M. Żukowski, Rev. Mod. Phys. 84, 777 (2012).

[3] S. Aaronson and A. Arkhipov, Proceedings of the 43rd Annual ACM Symposium on Theory of Computing, 2011, San Jose (ACM, New York, 2011), p. 333.

[4] P. Lodahl, S. Mahmoodian, and S. Stobbe, Rev. Mod. Phys. 87, 347 (2015).

[5] P. Michler et al., Science 290, 2282 (2000); C. Santori, M. Pelton, G. Solomon, Y. Dale, and Y. Yamamoto, Phys. Rev. Lett. 86, 1502 (2001); Z. Yuan, B. E. Kardynal, R. M. Stevenson, A. J. Shields, C. J. Lobo, K. Cooper, N. S. Beattie, D. A. Ritchie, and M. Pepper, Science 295, 102 (2002).

[6] J. M. Gérard, B. Sermage, B. Gayral, B. Legrand, E. Costard, and V. Thierry-Mieg, Phys. Rev. Lett. 81, 1110 (1998); M. Pelton, C. Santori, J. Vučković, B. Zhang, G. S. Solomon, J. Plant, and Y. Yamamoto, Phys. Rev. Lett. 89, 233602 (2002); S. Strauf, N. G. Stoltz, M. T. Rakher, L. A. Coldren, P. M. Petroff, and D. Bouwmeester, Nat. Photonics 1, 704 (2007); T. Heindel, C. Schneider, M. Lermer, S. H. Kwon, T. Braun, S. Reitzenstein, S. Höfling, M. Kamp, and A. Forchel, Appl. Phys. Lett. 96, 011107 (2010).

[7] D. Englund, D. Fattal, E. Waks, G. Solomon, B. Zhang, T. Nakaoka, Y. Arakawa, Y. Yamamoto, and J. Vučković, Phys. Rev. Lett. 95, 013904 (2005); A. Faraon, I. Fushman, D. Englund, N. Stoltz, P. Petroff, and J. Vuckovic, Nat. Phys. 4, 859 (2008); K. H. Madsen, S. Ates, J. Liu, A. Javadi, S. M. Albrecht, I. Yeo, S. Stobbe, and P. Lodahl, Phys. Rev. B 90, 155303 (2014); L. Sapienza, M. Davanco, A. Badolata, and K. Srinivasan, Nat. Commun. 6, 7833 (2015).

[8] J. Claudon et al., Nat. Photonics 4, 174 (2010); M.E. Reimer, G. Bulgarini, N. Akopian, M. Hocevar, M. B. Bavinck, M. A. Verheijen, E. P. A. M. Bakkers, L. P. Kouwenhoven, and V. Zwiller, Nat. Commun. 3, 737 (2012); M. Munsch, N. S. Malik, E. Dupuy, A. Delga, J. Bleuse, J.-M. Gérard, J. Claudon, N. Gregersen, and J. Mørk, Phys. Rev. Lett. 110, 177402 (2013).
[9] M. Gschrey et al., Nat. Commun. 6, 7662 (2015); A. Thoma et al., arXiv:1507.05900.

[10] C. Santori, D. Fattal, J. Vučković, G. S. Solomon, and Y. Yamamoto, Nature (London) 419, 594 (2002); O. Gazzano, S. Michaelis de Vasconcellos, C. Arnold, A. Nowak, E. Galopin, I. Sagnes, L. Lanco, A. Lemaître, and P. Senellart, Nat. Commun. 4, 1425 (2013); P. Gold, A. Thoma, S. Maier, S. Reitzenstein, C. Schneider, S. Höfling, and M. Kamp, Phys. Rev. B 89, 035313 (2014); S. Maier, P. Gold, A. Forchel, N. Gregersen, J. Mørk, S. Höfling, C. Schneider, and M. Kamp, Opt. Express 22, 8136 (2014); S. Unsleber, D. P. S. McCutcheon, M. Dambach, M. Lermer, N. Gregersen, S. Höfling, J. Mørk, C. Schneider, and M. Kamp, Phys. Rev. B 91, 075413 (2015).

[11] Y.-M. He, Y. He, Y.-J. Wei, D. Wu, M. Atatüre, C. Schneider, S. Höfling, M. Kamp, C.-Y. Lu, and J.-W. Pan, Nat. Nanotechnol. 8, 213 (2013); Y.-J. Wei et al., Nano Lett. 14, 6515 (2014).

[12] Y. He et al., Phys. Rev. Lett. 111, 237403 (2013); T. M. Sweeney et al., Nat. Photonics 8, 442 (2014).

[13] V. Giesz, O. Gazzano, A. K. Nowak, S. L. Portalupi, A. Lemaître, I. Sagnes, L. Lanco, and P. Senellart, Appl. Phys. Lett. 103, 033113 (2013).

[14] A. J. Bennett, D. C. Unitt, A. J. Shields, P. Atkinson, and D. A. Ritchie, Opt. Express 13, 7772 (2005); E. B. Flagg, A. Muller, S. Polyakov, A. Ling, A. Migdall, and G. Solomon, Phys. Rev. Lett. 104, 137401 (2010).

[15] C. Santori, D. Fattal, J. Vuckovic, G. S. Solomon, and Y. Yamamoto, New J. Phys. 6, 89 (2004).

[16] A. Müller, E. B. Flagg, P. Bianucci, X. Y. Wang, D. G. Deppe, W. Ma, J. Zhang, G. J. Salamo, M. Xiao, and C. K. Shih, Phys. Rev. Lett. 99, 187402 (2007); A. N. Vamivakas, Y. Zhao, C.-Y. Lu, and M. Atatüre, Nat. Phys. 5, 198 (2009); E. B. Flagg, A. Muller, J. W. Robertson, S. Founta, D. G. Deppe, M. Xiao, W. Ma, G. J. Salamo, and C. K. Shih, Nat. Phys. 5, 203 (2009); S. Ates, S. M. Ulrich, S. Reitzenstein, A. Löffler, A. Forchel, and P. Michler, Phys. Rev. Lett. 103, 167402 (2009); D. Englund, A. Majumdar, A. Faraon, M. Toishi, N. Stoltz, P. Petroff, and J. Vučković, Phys. Rev. Lett. 104, 073904 (2010).

[17] H. Jayakumar, A. Predojević, T. Huber, T. Kauten, G. S. Solomon, and G. Weihs, Phys. Rev. Lett. 110, 135505 (2013); M. Müller, S. Bounouar, K. D. Jöns, M. Glässl, and P. Michler, Nat. Photonics 8, 224 (2014).

[18] S. Unsleber et al., arXiv:1512.01048 [Opt. Express (to be published)].

[19] M. Fox, Quantum Optics (Oxford University Press, New York, 2006).

[20] J. Houel et al., Phys. Rev. Lett. 108, 107401 (2012).

[21] A. Zrenner, E. Beham, S. Stufler, F. Findeis, M. Bichler, and G. Abstreiter, Nature (London) 418, 612 (2002); R. Melet, V. Voliotis, A. Enderlin, D. Roditchev, X. L. Wang, T. Guillet, and R. Grousson, Phys. Rev. B 78, 073301 (2008).

[22] R. Hanbury Brown and R. Q. Twiss, Nature (London) 178, 1447 (1956).

[23] M. A. Broome, A. Fedrizzi, S. Rahimi-Keshari, J. Dove, S. Aaronson, T. C. Ralph, and A. G. White, Science 339, 794 (2013); J. B. Spring et al., Science 339, 798 (2013); M. Tillmann, B. Dakić, R. Heilmann, S. Nolte, A. Szameit, and P. Walther, Nat. Photonics 7, 540 (2013); A. Crespi, 
R. Osellame, R. Ramponi, D. J. Brod, E. F. Galvão, N. Spagnolo, C. Vitelli, E. Maiorino, P. Mataloni, and F. Sciarrino, Nat. Photonics 7, 545 (2013).

[24] A. Delteil et al., arXiv:1507.00465.

[25] C. K. Hong, Z. Y. Ou, and L. Mandel, Phys. Rev. Lett. 59, 2044 (1987).

[26] P. C. Humphreys, B. J. Metcalf, J. B. Spring, M. Moore, X.-M. Jin, M. Barbieri, W. Steven Kolthammer, and I. A. Walmsley, Phys. Rev. Lett. 111, 150501 (2013); K. R. Motes, A. Gilchrist, J. P. Dowling, and P. P. Rohde, Phys. Rev. Lett. 113, 120501 (2014).

[27] P. G. Kwiat, K. Mattle, H. Weinfurter, A. Zeilinger, A. V. Sergienko, and Y. Shih, Phys. Rev. Lett. 75, 4337 (1995).
[28] X.-C. Yao, T.-X. Wang, P. Xu, H. Lu, G.-S. Pan, X.-H. Bao, C.-Z. Peng, C.-Y. Lu, Y.-A. Chen, and J.-W. Pan, Nat. Photonics 6, 225 (2012).

[29] C.-Y. Lu, Y. Zhao, A. N. Vamivakas, C. Matthiesen, S. Fält, A. Badolato, and M. Atatüre, Phys. Rev. B 81, 035332 (2010); A. N. Vamivakas, C.-Y. Lu, C. Matthiesen, Y. Zhao, S. Fält, A. Badolato, and M. Atatüre, Nature (London) 467, 297 (2010); A. Delteil, W.-b. Gao, P. Fallahi, J. MiguelSanchez, and A. Imamoğlu, Phys. Rev. Lett. 112, 116802 (2014).

[30] F. Marsili et al., Nat. Photonics 7, 210 (2013).

[31] N. Somaschi et al., arXiv:1510.06499. 